\title{
Transparency in our decision making
}

\author{
By removing ambiguity and opacity in how we communicate with authors and by striving for timely \\ and efficient handling of all manuscripts, Nature Microbiology is committed to providing a high-quality \\ experience for all researchers submitting work for consideration, whether selected for publication or not.
}

Acronyms can provide a useful tool for abbreviating complex names or phrases, allowing faster communication, whether in writing or conversation. However, the utility of a TLA (Box 1) breaks down if the intended meaning of the abbreviation is unclear to any party involved in the discourse. The ambiguity created can lead to misunderstanding and confusion; AFAIK, IRL PPL say "OMG" not "THX" for overuse of abbreviations.

ICYMI, scientific publishing is a veritable minefield of acronyms, whether in shortening lengthy names for journals (for example, $\mathrm{CH} \& \mathrm{M}$ and ISMEJ), organisms (for example, DENV and LUCA) or genes/proteins (for example, fts $Z$ and CRP). Another area replete with myriad acronyms has been the description of the publishing process. Different pockets of the scientific journal landscape employ a diverse and often bewildering array of acronyms to describe various stages, from submission of a manuscript through eventual rejection or publication. Even within a single group of journals (the NRJs, for example), the use and meaning of acronyms employed can vary from one journal to the next. For example, the action of rejecting a manuscript without sending it out for review has been variably labelled as RTA, RTA-NR or $\mathrm{OE}$, depending on the journal. In the latter case, $\mathrm{OE}$ isn't even an acronym but rather derives from an instruction to add stock paragraphs $\mathrm{O}$ and $\mathrm{E}$ to a handwritten note from the editor when decision letters were being produced on typewriter in the pre-PC era. Other abbreviations may be used in a more standard fashion across a journal group (OD, DC, P\&Q, AIP and $\mathrm{AOP}$ ), but their meanings are not necessarily any clearer for authors, and this is why the use of such acronyms has become a problem. Precision should be fundamental to scientific communication, and we acknowledge that the ambiguity caused by labelling decisions in this way can be confusing to some authors, who are able to see these notations when logged in to our system. Consequently, as part of a wider drive to improve transparency in decisionmaking processes, Nature Microbiology and our sister titles have recently adopted new ratings based on just three terms (reject, revise or accept). Hopefully, this will make the status of a manuscript clearer to authors. There will be variations for each type of rating depending on the stage of the manuscript (before review/postreview) and the manuscript type, but the intention behind each decision should now be unambiguous.

Updating the terminology we use to describe our decision-making is just one way in which we are striving to ensure open and transparent communication with authors. Since Nature Microbiology opened for submissions in May 2015, rather than relying solely on form-letters, we have been providing tailored decisions for authors at each stage of submission, outlining the specific reasoning behind our decisions. Whether it's a pre-submission enquiry or a full manuscript, if we do not invite full submission or send a manuscript out

\section{Box 1 | Glossary of acronyms.}

AFAIK: as far as I know

AIP: accept in principle

AOP: advance online publication

BTW: by the way

CH\&M: Cell Host \& Microbe

CRP: cyclic AMP (cAMP) receptor protein

DC: door closed

DENV: dengue virus

ftsZ: filamenting temperature-sensitive mutant $Z$

ICYMI: in case you missed it

IRL: in real life

ISMEJ: International Society for Microbial Ecology Journal

LUCA: last universal common ancestor

NRJ: Nature research journal

OD: open door

OMG: oh my god

P\&Q: pending quotations

PC: personal computer

PPL: people

RTA: return to author

RTA-NR: return to author no review

THX: thanks

TLA: three letter acronym

TTFN: ta ta for now for review (that is, reject), we explain the reasoning behind this editorial decision, giving a clear indication of what we felt was missing, or which previously published studies affected the strength of the conceptual advance provided. This not only helps authors to better understand what makes a potential Nature Microbiology paper, but also enables authors to see whether a key aspect of the work might have been underappreciated (we editors are by no means infallible, BTW), in which case an appeal might be in order. Indeed, by signing each decision letter and including editors' contact details - which can also be found on our webpage (http://www.nature.com/ nmicrobiol/about/editors) - we ensure that authors can get in touch to ask us about any aspect of the decision process.

How, then, do we assess a submission? Each editor on Nature Microbiology handles work mainly from a few specific sub-disciplines, although manuscripts will sometimes be shared around the rest of the team to cover vacations and busy periods. Despite a common myth to the contrary, each editor carefully reads through an entire manuscript and associated material, looking closely at the data, the techniques used and the strength of support for the main conclusions, and writes comprehensive notes to circulate to colleagues. Our editors also assess the degree of conceptual advance by comparing each manuscript against the previously published literature. There is no single golden rule for what makes a paper suitable for consideration, but we are looking to publish papers that provide a substantial advance on the previous state of the field in a number of areas, including (but not necessarily limited to): breakthrough value, conceptual novelty, mechanistic insight, translational potential and resource value. What we do not consider is who the authors are, or which institutions they work at. Neither of these factor into whether or not we will send a piece of research out for review. Most manuscripts will likely be seen by at least two editors, who will discuss the relative merits of the manuscript and the best course of action, whether that is to have a manuscript peer-reviewed, or to reject editorially. 
Where a manuscript is selected to be sent out for review, the editor will then recruit a panel of referees with complementary expertise to make sure that the biological and technical aspects of a manuscript can all be suitably assessed. We look for referees with a range of experience, including senior researchers and those in the earlier stages of their careers, and we actively encourage the inclusion of junior people in referees' laboratories to help with their training (although not as a way of delegating the review process altogether). Once all of the referee reports have been received, our editors then read through them carefully, taking note of the referees' overall assessment of the manuscript, which points raised are fundamental to address should the paper be considered further for publication, and whether any requests are potentially unreasonable or out of scope for the current work. In forming our overall decision, we also consider the likely time needed to address the issues raised with new experimentation and revisions to the text. As with pre-review decisions, we then craft a personalized decision letter outlining the main points raised by the referees that form the reasoning behind our decision, and provide any specific editorial guidance if a revision is invited.

Increasing transparency on how we formulate and communicate decisions on manuscripts is just one of the ways in which we are exploring how we best serve our authors and the wider research community. Do please tell us what you think we are getting right, what we are getting wrong, and how we can better work with you to ensure that our journal represents the leading research, ideas and practices in microbiology today. TTFN. 